\title{
Estudo comparativo da ultrafiltração convencional eassociação de ultrafiltração convencional e modificada na correção de cardiopatias congênitas com alto risco cirúrgico
}

\author{
Miguel Angel MALUF*, Cristina MANGIA*, João BERTUCCEZ*, Célia SILVA*, Roberto CATANI*, \\ Werther CARVALHO*, Antônio CARVALHO*, Énio BUFFOLO*
}

RBCCV 44205-464

Maluf MA, Mangia C, Bertuccez J, Silva C, Catani R, Carvalho W, Carvalho A, Buffolo E - Estudo comparativo da ultrafiltração convencional e associação de ultrafiltração convencional e modificada na correção de cardiopatias congênitas com alto risco cirúrgico. Rev Bras Cir Cardiovasc 1999; 14(3): 221-36

RESUMO: A necessidade de correção cirúrgica de má-formações cardíacas complexas, que requerem tempos prolongados de circulação extracorpórea (CEC) aumentou a morbimortalidade devido a retenção hídrica e reação inflamatória sistêmica. O objetivo deste estudo é comparar a evolução pós-operatória imediata de pacientes submetidos a ultrafiltração convencional (UFC) durante a CEC e ultrafiltração modificada (UFM) após CEC. Quarenta e um pacientes submetidos a correção cirúrgica de cardiopatias congênitas foram divididos em 2 grupos: G1: 21 pacientes com idade de 15 dias a 36 meses (mediana: 11 meses) e peso de 3,6 a 13,5 kg (M: 7,27 $\pm 3,07)$, operados entre 1996 e 1997, foram submetidos a UFC. G2: 20 pacientes com idade de 9 dias a 36 meses (mediana: 5,5 meses) e peso entre 2,2 e $12 \mathrm{~kg}(\mathrm{M}: 5,7 \pm 2,5)$, operados entre 1997 e 1998, foram submetidos a UFC+UFM. Dentre as operações mais freqüentes temos: ventriculosseptoplastia, $15(36,5 \%)$ casos; operação de Jatene, $10(24,3 \%)$ casos; correção de defeito septal A-V total, $7(17,0 \%)$ casos etc. A análise estatística de idade, peso e complexidade cirúrgica mostrou semelhança entre os grupos. Houve $6(28,5 \%)$ óbitos no $\mathrm{G} 1$ e $4(20 \%)$ no $G 2$, $(p=0,71)$. O volume médio ultrafiltrado no G1 (UFC) foi 143,3 ml e no G2 (UFC+UFM) foi 227,0 ml, $(p<0,001$ ), mostrando diferença estatisticamente significante. Porém o tempo médio de ventilação mecânica ( $G 1: 94$.8h, $G 2: 95.6 h, p=0.97)$, tempo médio de uso de drogas inotrópicas ( $G 1: 145.2 \mathrm{~h}$ e $\mathrm{G} 2: 137.6 \mathrm{~h}, \mathrm{p}=0.85)$; tempo médio de permanência em UTIP (G1: 169.6h e G2: 157.8h, p= 0.79) e tempo médio de permanência hospitalar (G1: 14,8 d. e G2 $14,6$ d., $p=0,95)$, não mostraram diferenças significantes entre os grupos. A técnica de UFC utilizada a mais de 8 anos no nosso Serviço mostrou resultados semelhantes quando comparada com a associação de UFC+UFM. A UFM mostrou-se eficaz na remoção de água corpórea após CEC, não havendo intercorrências com o método. Talvez um estudo randomizado, recrutando um número maior de pacientes permita detectar diferenças entre os dois métodos.

DESCRITORES: Cardiopatias congênitas, cirurgia. Hemofiltração, métodos. Circulação extracorpórea.

Trabalho realizado na Disciplina de Cirurgia Cardiovascular da Escola Paulista de Medicina. Universidade Federal de São Paulo. São Paulo, SP, Brasil. Apresentado ao 26ํㅡㄹ Congresso Nacional de Cirurgia Cardíaca, Fortaleza, CE, 8 a10 de abril, 1999.

* Da Escola Paulista de Medicina da Universidade Federal de São Paulo.

Endereço para correspondência: Miguel A. Maluf. Rua Apeninos, 930, Conj. 182, Paraíso. São Paulo, SP, Brasil. CEP: $04104-020$. Tel: (011) 539-7725. e-mail:miguel@bes-way.com.br. 
Maluf MA, Mangia C, Bertuccez J, Silva C, Catani R, Carvalho W, Carvalho A, Buffolo E - Estudo comparativo da ultrafiltração convencional e associação de ultrafiltração convencional e modificada na correção de cardiopatias congênitas com alto risco cirúrgico. Rev Bras Cir Cardiovasc 1999; 14 (3): 221-36

\section{INTRODUÇÃO}

Apesar da evolução dos conhecimentos e da melhora tecnológica na área de cirurgia cardíaca, o uso da circulação extracorpórea (CEC), na correção de cardiopatias congênitas em neonatos e lactentes, constitui um grande desafio.

Ainda não foi possível contar com equipamento ideal que dispense o uso de sangue no perfusato, evite a retenção hídrica e não comprometa as funções orgânicas. Numerosas técnicas têm sido desenvolvidas para reverter o edema tissular e a hemodiluição após CEC, como: hemofiltração durante a CEC, uso de diuréticos, diálise peritoneal e hemofiltração artério-venosa contínua no pós-operatório.

A hemofiltração ou ultrafiltração são procedimentos cujo objetivo principal é a retirada de água e substâncias de baixo peso molecular do plasma, que se encontram submetidas a gradiente de pressão hidrostática. Para tal fim são empregadas membranas semipermeáveis semelhantes às utilizadas nos procedimentos de hemodiálise.

O primeiro ultrafiltrador foi desenvolvido por BRULL em 1928(1) e posteriormente aperfeiçoado por ALWALL (2) e LUNDERQUIST(3), empregandose, na época, tubos de celofane como membrana de filtração. Foi este último quem realizou as primeiras aplicações clínicas do método em seres humanos, para o tratamento de edema refratário em pacientes com insuficiência renal.

Duas décadas se passaram até que o emprego da ultrafiltração em cirurgia cardíaca fosse relatado por ROMAGNOLI et al. (4), DARUP et al. (5), e MAGILIGAN Jr. ${ }^{(6)}$, na tentativa de reverter a hemodiluição da CEC, ou no período pós-operatório (NAIK et al.(7)).

A ultrafiltração durante a CEC tinha como principal desvantagem o pequeno volume de perfusato utilizado em crianças; já a ultrafiltração durante o pós-operatório muitas vezes não era possível, em razão da instabilidade hemodinâmica do paciente.

A técnica de ultrafiltração em crianças foi aperfeiçoada e posteriormente modificada por Elliot (NAIK et al. ${ }^{(8)}$ ). Num estudo piloto, foi mudada a posição do ultrafiltrador dentro do circuito da CEC e o momento do seu uso.

Desta maneira, dois métodos diferentes de ultrafiltração foram estabelecidos:

- A ultrafiltração durante a CEC é chamada de ultrafiltração convencional (UFC). Neste modelo, a ultrafiltração do perfusato é realizada enquanto 0 paciente se encontra assistido pela máquina coração-pulmão.

- A ultrafiltração realizada depois que o paciente foi desligado da CEC é chamada de ultrafiltração modificada (UFM). Neste modelo é ultrafiltrado apenas o sangue do paciente e também hemoconcentrado o perfusato, devolvendo ao paciente todas as hemácias retiradas do circuito.

A ultrafiltração na CEC tem como principais objetivos:

- Remover o excesso de água corporal

- Reduzir o uso de transfusões sangüíneas durante o pós-operatório

- Melhorar a função orgânica após CEC

- Filtrar os mediadores responsáveis pela reação inflamatória generalizada.

O emprego da CEC em crianças submetidas a correção de defeitos congênitos foi responsável por morbimortalidade não desprezível na Disciplina de Cirurgia Cardiovascular do Departamento de Cirurgia da EPM da UNIFESP, nos últimos 10 anos. A cuidadosa observação da evolução pós-operatória destes pacientes e a necessidade de intervir em faixas etárias menores ou com cardiopatias complexas nos obrigaram a introduzir algumas mudanças na CEC, como composição e volume do perfusato, composição e métodos de proteção miocárdica, utilização de oxigenadores de membrana, cânulas e circuitos apropriados e emprego da UFC.

Apesar das mudanças na tática e técnica cirúrgica e modificações introduzidas na CEC, os resultados obtidos não atenderam as nossas expectativas.

A procura constante na melhora dos resultados nos motivou a introduzir a UFM, com especial indicação nas operações de pacientes neonatos, lactentes e infantes portadores da cardiopatias complexas, que necessitavam tempos prolongados de CEC para sua correção cirúrgica.

O objetivo deste estudo é comparar a evolução pós-operatória imediata de pacientes submetidos a UFC e associação de UFC+UFM.

\section{CASUÍSTICA E MÉTODOS}

\section{a) Descrição da Casuística}

De janeiro de 1996 a março de 1998, foram operados 280 pacientes portadores de cardiopatias congênitas, na EPM-UNIFESP. Quarenta e um (14,6\%) pacientes deste grupo, menores de 36 meses, portadores de cardiopatia congênita de moderada a grave complexidade cirúrgica e operados com auxílio da CEC, foram divididos em dois grupos e analisados conforme o método de ultrafiltração utilizado (MALUF ${ }^{(9)}$ ). 
Maluf MA, Mangia C, Bertuccez J, Silva C, Catani R, Carvalho W, Carvalho A, Buffolo E - Estudo comparativo da ultrafiltração convencional e associação de ultrafiltração convencional e modificada na correção de cardiopatias congênitas com alto risco cirúrgico. Rev Bras Cir Cardiovasc 1999; 14 (3): 221-36

O Grupo I foi constituído por 21 pacientes, 10 do sexo masculino e 11 do sexo feminino, com idades que variaram entre 15 dias e 36 meses (mediana: 11 meses) e peso oscilando entre 3,6 e $13,5 \mathrm{~kg}$ (média: $7,27 \pm 3,07)$. Quatorze $(66,7 \%)$ pacientes eram portadores de cardiopatias acianogênicas e 7 $(33,3 \%)$ pacientes possuíam cardiopatias cianogênicas. Nenhum paciente tinha sido submetido a operações prévias. Neste grupo de pacientes, operados entre janeiro de 1996 e maio de 1997, foi utilizada a ultrafiltração convencional (UFC), durante a CEC (Quadro 1).

O Grupo II, foi formado por 20 pacientes, 10 do sexo masculino e 10 do sexo feminino, com idades que variaram entre 9 dias e 36 meses (mediana: 5,5 meses) e peso oscilando entre 2,2 e $12 \mathrm{~kg}$ (média: $5,77 \pm 2,54)$. Onze $(55 \%)$ pacientes eram portadores de cardiopatias acianogênicas e 9 (45\%) pacientes eram portadores de cardiopatias cianogênicas. Apenas $2(10 \%)$ pacientes tinham sido submetidos a operações prévias: caso № 5 , bandagem pulmonar caso № 17, operação de Blalock-Taussig (B-T). Neste grupo de pacientes operados entre junho de 1997 e março de 1998, foi utilizada a ultrafiltração convencional associada a ultrafiltração modificada. (UFC+UFM) (Quadro 2).

\section{b) Grau de Complexidade da Correção Cirúrgica}

Os pacientes de cada grupo foram classificados conforme o grau de complexidade da correção cirúrgica, seguindo os conceitos de JENKINS et al.(10), que estabeleceram 4 graus de complexidade, segundo a gravidade da correção cirúrgica. No grupo I, 16 $(76,1 \%)$ pacientes tinham grau 2 de complexidade cirúrgica; 1 (4,76\%) caso, grau 3 e $4(19,0 \%)$ casos, grau 4 (Tabela 3 ).

No grupo II, $10(50 \%)$ pacientes tinham grau 2 de complexidade cirúrgica; 4 (20\%) casos, grau 3 e $6(30 \%)$ casos, grau 4 (Tabela 4$)$.

\section{c) Técnica de CEC}

Foram utilizados oxigenadores pediátricos, constituídos por membrana capilar de fibras ocas ("microporous polypropylene"), com fluxo externo de sangue e $\mathrm{O}_{2}$ e ar comprimido, pela parede interna (Oxim II, Macchi ${ }^{\circledR}$ e Dideco ${ }^{\circledR}$ Mirandola, Itália).

Em crianças de até $5 \mathrm{~kg}$, foi utilizada linha arterial de $3 / 16$ polegadas e venosa de $1 / 4$ polegadas e, em crianças de 5 a $12 \mathrm{~kg}$, foi empregada linha arterial e venosa de $1 / 4$ polegadas.

QUADRO 1

DADOS DOS PACIENTES E TIPO DE CORREÇÃO CIRÚRGICA

GRUPO I - ULTRAFILTRAÇÃO CONVENCIONAL (UFC)

\begin{tabular}{|c|c|c|c|c|c|}
\hline $\begin{array}{l}\text { PACIENTE } \\
\text { № ORDEM }\end{array}$ & $\begin{array}{c}\text { IDADE } \\
\text { (MESES) }\end{array}$ & SEXO & $\begin{array}{c}\text { PESO } \\
\text { (kg) }\end{array}$ & DIAGNÓSTICO & TIPO DE OPERAÇÃO \\
\hline 1 & 5 & M & 5,70 & $\mathrm{TGA}+\mathrm{CIA}$ & Correção total (op. Jatene) \\
\hline 2 & 10 & $\mathrm{~F}$ & 4,50 & $\mathrm{CIV}+\mathrm{HP}$ & Correção total (ventriculosseptoplastia) \\
\hline 3 & 6 & $\mathrm{~F}$ & 4,70 & D.S.A - VT + IM + HP & Correção total (duplo retalho + plástica mitral) \\
\hline 4 & 7 & $\mathrm{~F}$ & 4,00 & D.S.A. $-V T+P C A+H P$ & Correção total (duplo retalho + ligadura de PCA) \\
\hline 5 & 5 & M & 5,30 & D.S.A. $-V T+H P$ & Correção total (duplo retalho) \\
\hline 6 & 7 & $\mathrm{~F}$ & 5,00 & DVSVD + CIV SUB PUL + HP & Correção total (op. Jatene) \\
\hline 7 & 8 & M & 6,00 & $T G A+C I A$ & Correção total (op. Jatene) \\
\hline 8 & 8 & M & 8,00 & Tétrade de Fallot & Correção total (transatrial e pulmonar) \\
\hline 9 & 2 & $\mathrm{~F}$ & 3,60 & DATVP + HP & Correção total (ampliação do AE) \\
\hline 10 & 20 & $\mathrm{~F}$ & 8,70 & $\mathrm{CIV}+\mathrm{HP}$ & Correção total (ventriculosseptoplastia) \\
\hline 11 & 21 & M & 7,50 & D. S. A. $-V T+H P+$ S.Down & Correção total (duplo retalho) \\
\hline 12 & 32 & $\mathrm{~F}$ & 11,00 & $\mathrm{CIV}+\mathrm{PCA}+\mathrm{HP}$ & Correção total (ventriculosseptoplastia + lig. PCA) \\
\hline 13 & 13 & M & 7,60 & $\mathrm{CIV}+\mathrm{HP}$ & Correção total (ventriculosseptoplastia) \\
\hline 14 & 24 & $\mathrm{~F}$ & 10,70 & $\mathrm{CIV}+\mathrm{CIA}+\mathrm{PCA}+\mathrm{HP}$ & Correção total (átrio - ventriculosseptoplastia + lig. PCA) \\
\hline 15 & 36 & M & 13,50 & Tétrade de Fallot & Correção total (reconstrução VSVD C/P bivalvular) \\
\hline 16 & $15 / 30$ & M & 3,70 & $T G A+C I A$ & Correção total (op. Jatene) \\
\hline 17 & 36 & M & 11,20 & $\mathrm{CIV}+\mathrm{IM}+\mathrm{HP}$ & Correção total (ventriculosseptoplastia + plástica mitral) \\
\hline 18 & 35 & $\mathrm{~F}$ & 8,80 & $\mathrm{ClA}+\mathrm{PCA}+\mathrm{HP}$ & Correção total (atriosseptoplastia + lig. PCA) \\
\hline 19 & 5 & $\mathrm{~F}$ & 4,00 & $\mathrm{CIV}+\mathrm{PCA}+\mathrm{HP}$ & Correção total (ventriculosseptoplastia + lig. PCA) \\
\hline 20 & 12 & M & 6,65 & $\mathrm{CIV}+\mathrm{PCA}+\mathrm{HP}$ & Correção total (ventriculosseptoplastia + lig. PCA) \\
\hline 21 & 24 & $\mathrm{~F}$ & 12,60 & Origem Anômala de ACE & Correção total (reimplante da ACE na AO) \\
\hline
\end{tabular}


Maluf MA, Mangia C, Bertuccez J, Silva C, Catani R, Carvalho W, Carvalho A, Buffolo E - Estudo comparativo da ultrafiltração convencional e associação de ultrafiltração convencional e modificada na correção de cardiopatias congênitas com alto risco cirúrgico. Rev Bras Cir Cardiovasc 1999; 14 (3): 221-36

QUADRO 2

DADOS DOS PACIENTES E TIPO DE CORREÇÃO CIRÚRGICA

GRUPO II - ULTRAFILTRAÇÃO CONVENCIONAL (UFC) + ULTRAFILTRAÇÃO MODIFICADA (UFM)

\begin{tabular}{|c|c|c|c|c|c|}
\hline $\begin{array}{l}\text { PACIENTE } \\
\text { № ORDEM }\end{array}$ & $\begin{array}{c}\begin{array}{l}\text { IDADE } \\
\text { (MESES) }\end{array}\end{array}$ & SEXO & $\begin{array}{l}\text { PESO } \\
\text { (kg) }\end{array}$ & DIAGNÓSTICO & TIPO DE OPERAÇÃO \\
\hline 1 & 5 & $\mathrm{~F}$ & 3,50 & D.S.A. - VT + HP & Correção total (duplo retalho) \\
\hline 2 & 5 & $\mathrm{~F}$ & 4,85 & $\mathrm{CIV}+\mathrm{HP}$ & Correção total (ventriculosseptoplastia) \\
\hline 3 & 6 & M & 4,20 & $\mathrm{CIV}+\mathrm{HP}$ & Correção total (ventriculosseptoplastia) \\
\hline 4 & 5 & $\mathrm{~F}$ & 3,90 & DVSVD + CIV SUB PUL. + HP & Correção total (op. Jatene) \\
\hline 5 & 11 & M & 6,40 & TGA + CIV + BAND. PUL. & Correção total (op. Jatene) \\
\hline 6 & 2 & M & 3,30 & COR TRIATRIATUM + HP & Correção total (ressecção membrana) \\
\hline 7 & 5 & M & 5,80 & $\mathrm{TGA}+\mathrm{CIA}+\mathrm{HP}$ & Correção parcial (op. Jatene + septectomia atrial) \\
\hline 8 & 9 & M & 7,00 & $\mathrm{CIV}+\mathrm{HP}$ & Correção total (ventriculosseptoplastia) \\
\hline 9 & 11 & $\mathrm{~F}$ & 7,00 & D.S.A - VT + IM + HP & Correção total (duplo retalho + plástica mitral) \\
\hline 10 & 11 & M & 4,50 & TRUNCUS ARTERIOSUS TIPO I & Correção total (op. Barbero - Marcial) \\
\hline 11 & 5 & $\mathrm{~F}$ & 5,90 & D.S.A - VT + HP + S. Down & Correção total (duplo retalho) \\
\hline 12 & 5 & M & 5,00 & $\mathrm{CIV}+\mathrm{PCA}+\mathrm{HP}+\mathrm{S}$. Down & Correção total (ventriculosseptoplastia + lig. PCA) \\
\hline 13 & 17 & $\mathrm{~F}$ & 6,00 & $\mathrm{CIV}+\mathrm{HP}$ & Correção total (ventriculosseptoplastia) \\
\hline 14 & 20 & M & 10,00 & EST. APD - APE + PCA & Correção total (ampliação APD - APE + lig. PCA) \\
\hline 15 & 14 & M & 8,00 & $\mathrm{CIV}+\mathrm{CIA}+\mathrm{HP}$ & Correção total (atrio-ventriculosseptoplastia) \\
\hline 16 & 36 & M & 12,00 & $\mathrm{CIV}+\mathrm{HP}$ & Correção total (ventriculosseptoplastia) \\
\hline 17 & 16 & $\mathrm{~F}$ & 9,25 & DVEVD + EPV + TGA & Correção parcial (op. hemi-Fontan) \\
\hline 18 & $9 / 30$ & $\mathrm{~F}$ & 2,20 & $T G A+C I A$ & Correção total (op. Jatene) \\
\hline 19 & $11 / 30$ & $\mathrm{~F}$ & 3,10 & $\mathrm{TGA}+\mathrm{CIA}$ & Correção total (op. Jatene) \\
\hline 20 & $15 / 30$ & $\mathrm{~F}$ & 3,50 & $T G A+C I A$ & Correção total (op. Jatene) \\
\hline
\end{tabular}

Para o preenchimento do circuito extracorpóreo foi necessário um volume de 450 a $500 \mathrm{ml}$ de perfusato. A composição do perfusato dependeu das condições do paciente em relação ao peso, superfície corpórea e hematócrito $(\mathrm{Ht})$. A proporção de diluente e hemácias no perfusato foi suficiente para manter o $\mathrm{Ht}$ em torno de $25 \%$ durante a CEC, mediante a aplicação da fórmula de carga sangüínea de KIRKLIN \& BARRATT-BOYES ${ }^{(11)}$.

\begin{tabular}{c} 
Carga sangüínea $=$ \\
$\frac{\left[\begin{array}{l}\text { Ht desejado }(\mathrm{CEC})] \times\left[\text { volemia do paciente }^{*}+\text { volume }\right. \\
\text { do perfusato] }-\left[\text { volemia do paciente }{ }^{*} \times \mathrm{Ht} \text { do paciente] }\right.\end{array}\right.}{\mathrm{Ht} \text { bolsa }}$ \\
\hline
\end{tabular}

*Peso do paciente $\mathrm{x} 80$.

A composição do perfusato utilizado foi:

- Concentrado de hemácias (até 48h de coleta) e solução glicofisiológica ou plasma fresco (conforme fórmula)

- Manitol $20 \%$ - 2,0 $\mathrm{ml} / \mathrm{kg}$

- Bicarbonato de sódio $\left(\mathrm{NaHCO}_{3}\right)-10 \%$ - conforme fórmula: $(1,5 \times$ volume perfusato $\times \mathrm{BE})$ 1200

- Heparina (Liquemine) - $30 \mathrm{mg}(0,6 \mathrm{ml}) \times 500 \mathrm{ml}$ de sangue

- Kefasol: $50 \mathrm{mg} \times \mathrm{kg}$
A perfusão arterial foi realizada através de cânula posicionada na Ao imediatamente abaixo do tronco braquiocefálico e a drenagem venosa mediante cânulas posicionadas nas veias cavas. Em todos os casos foram descomprimidas as cavidades esquerdas, mediante a utilização de aspiração contínua de cateter ("vent"), introduzido na cavidade do ventrículo esquerdo, através da veia pulmonar superior direita.

Foi usado fluxo de perfusão estipulado em 2,2 a $2,4 \mathrm{l} / \mathrm{min} / \mathrm{m}^{2}$ de superfície corpórea, em condições de normotermia. Em todos os casos foi utilizada hipotermia moderada de 25 a $28^{\circ} \mathrm{C}$ e reduzido o fluxo de perfusão para 1,7 e $1,9 \mathrm{l} / \mathrm{min} / \mathrm{m}^{2}$.

O método da proteção miocárdica empregado, em todos os casos, foi a administração de solução cardioplégica sangüínea na porção inicial da aorta e através de hipotermia tópica, com infusão intermitente de solução salina gelada na cavidade pericárdica.

Composição da solução:

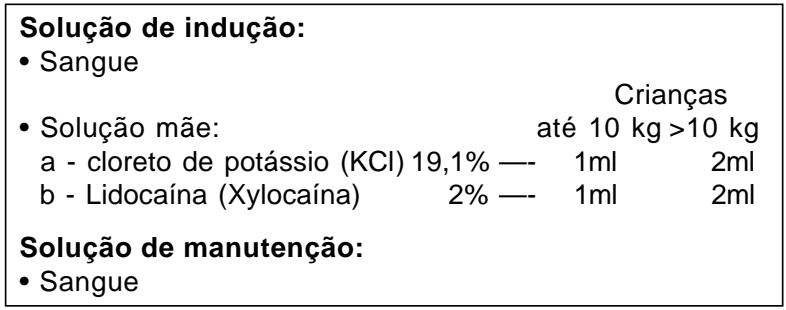


Maluf MA, Mangia C, Bertuccez J, Silva C, Catani R, Carvalho W, Carvalho A, Buffolo E - Estudo comparativo da ultrafiltração convencional e associação de ultrafiltração convencional e modificada na correção de cardiopatias congênitas com alto risco cirúrgico. Rev Bras Cir Cardiovasc 1999; 14 (3): 221-36

- Tempo de infusão: 2 a 3 min.

- Fluxo de infusão: $30 \mathrm{ml}$ x kg x min.

- Via de administração: foi utilizada a via anterógrada injetando a solução cardioplégica na aorta ou no próprio óstio coronariano.

- Temperatura: foi utilizado um permutador de calor para manter a temperatura da solução próxima aos $4^{\circ}$ a $6^{\circ} \mathrm{C}$

- Intervalo: as doses de manutenção foram repetidas a cada $20 \mathrm{~min}$.

- Reperfusão: o miocárdio foi reperfundido com sangue da linha arterial após despinçamento da aorta, ainda com o paciente em hipotermia.

\section{d) Técnica de Hemofiltração}

O sistema da hemofiltração foi montado no próprio circuito da cardioplegia (Macchi-Baxter ${ }^{\circledR}$ ) (Figura 1 ). Foi utilizado um hemoconcentrador, Hemocorplus HPH 400 (Minntech ${ }^{\circledR}$, Minneapolis, Minn.) (a), inserido em paralelo com a linha de cardioplegia (b) e o seu permutador de calor (c).

O circuito da solução cardioplégica (d) foi ligado diretamente à saída da recirculação do oxigenador, para se juntar com a saída do hemoconcentrador (a), e formar uma linha comum que passando pelo permutador de calor (c), terminava na própria linha de cardioplegia no campo cirúrgico.

Durante a CEC o circuito foi utilizado para a infusão de solução cardioplégica (d), mantendo-se ocluída a entrada do hemoconcentrador (e).

Após despinçamento da aorta e iniciado o aquecimento do paciente, o sangue do circuito de cardioplegia foi ocluído (f) e aberta a entrada (e) para o hemoconcentrador. Iniciou-se assim a UFC, conduzindo o sangue filtrado para a linha de recirculação (g) e drenando no reservatório de cardiotomia (h).

A UFM foi iniciada imediatamente após interromper a CEC, mantendo-se a cânula arterial (i) posicionada na aorta e ligada à linha arterial (j) e a cânula venosa $(\mathrm{k})$, posicionada na veia cava superior (VCS), agora recuada para o $A D$ e desligada da linha venosa (I) e ligada na linha da cardioplegia (d).

A UFM iniciou-se drenando sangue da linha arterial (j) mediante a rotação do rolete de cardioplegia (b) e conduzido para o hemoconcentrador (a). O sangue hemoconcentrado, após pinçamento da linha de recirculação $(m)$ foi orientado para o $A D$ pela linha de cardioplegia (d), passando pelo permutador de calor (c), que mantinha o sangue aquecido.

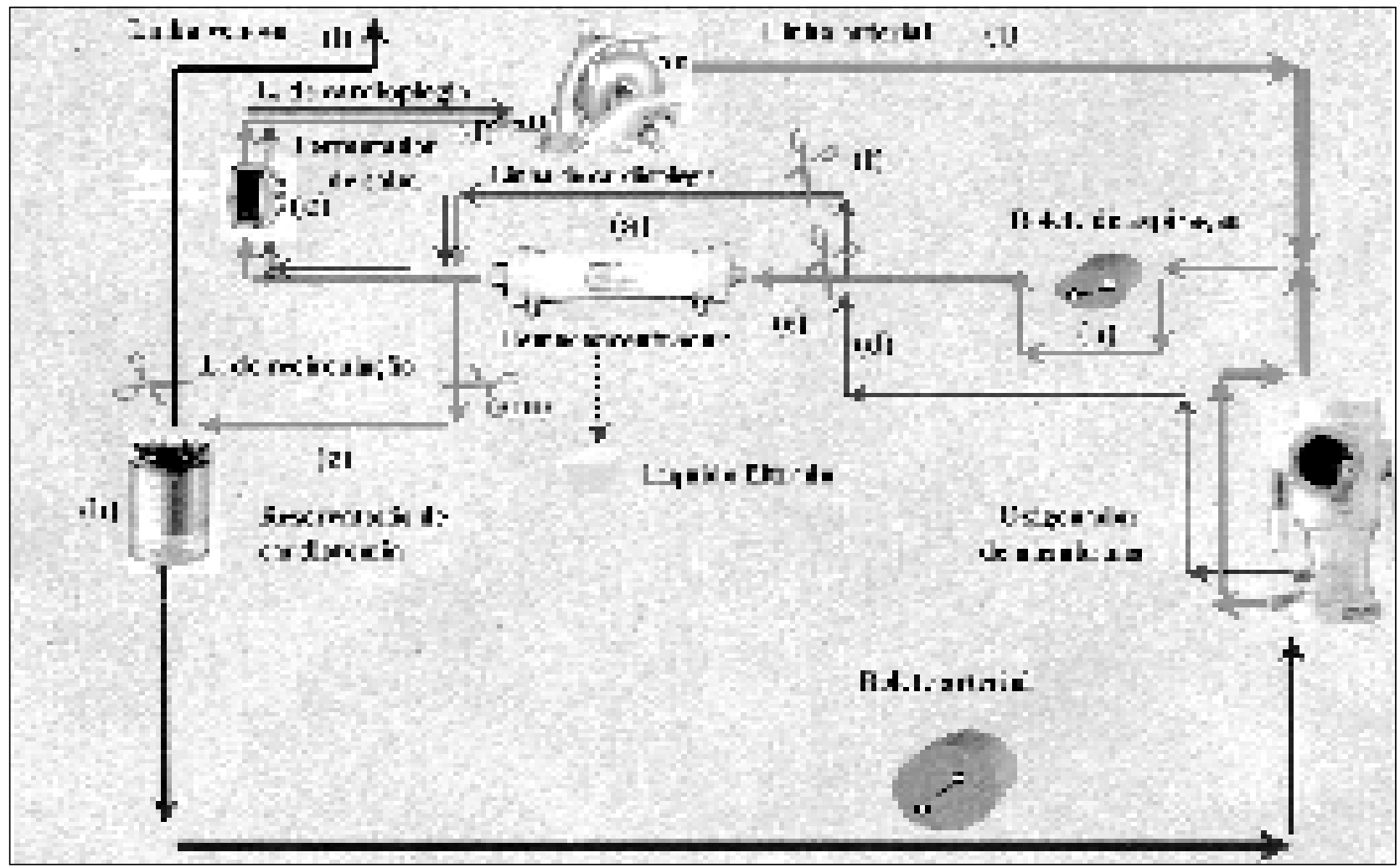

Fig. 1 - Circuito de ultrafiltração modificada no sistema de CEC. 
Maluf MA, Mangia C, Bertuccez J, Silva C, Catani R, Carvalho W, Carvalho A, Buffolo E - Estudo comparativo da ultrafiltração convencional e associação de ultrafiltração convencional e modificada na correção de cardiopatias congênitas com alto risco cirúrgico. Rev Bras Cir Cardiovasc 1999; 14 (3): 221-36

A utilização da bomba da cardioplegia permitiu manter um fluxo de filtração em torno de 100 a 300 $\mathrm{ml} / \mathrm{min}$. Não foi utilizada pressão negativa no hemoconcentrador para facilitar a filtração. Não foi registrada a pressão do circuito.

Simultaneamente ao processo de hemofiltração, foi possível a reposição volêmica do paciente, com controle das pressões arteriais e do AE.

A UFM foi encerrada quando o $\mathrm{Ht}$ do paciente atingiu $35 \%$ e todo o sangue remanescente no circuito foi filtrado e reinfundido no paciente.

O volume sangüíneo total (VST) foi definido como soma do volume do perfusato + a volemia estimada do paciente (NAIK et al. ${ }^{(8)}$ ). Foi calculado em todos os pacientes a relação volume ultrafiltrado/VST e no grupo II (UFM), o volume de ultrafiltração correspondeu à soma dos volumes de filtração durante a CEC e o volume obtido após CEC.

\section{e) Pós-operatório na Unidade de Terapia Intensiva Pediátrica}

A monitorização hemodinâmica constituiu em registro do $E C G$, pressão arterial, pressão do $A E$, pressão do TP. As drogas inotrópicas usadas foram: dopamina, dobutamina, adrenalina, amrinoma; drogas vasodilatadoras: nitroprussiato de sódio, nitroglicerina, tolazolina, prostaglandina $E_{1}$ etc.

Os pacientes portadores de defeitos cardíacos associados à HP podem apresentar no pós-operatório imediato (POI) elevação da pressão arterial pulmonar em forma de crise ou em forma contínua. A crise de HP foi definida como uma síndrome de elevação súbita da relação pressão pulmonar sistólica/pressão arterial sistólica (PPS/PAS) > 70\%, acompanhada de queda importante do débito cardíaco e $\mathrm{SvO}_{2}$. A HP persistente foi definida como a relação PPS/PAS $>70 \%$, contínua por período maior de 6h, após a correção cirúrgica.

Os pacientes que evoluíram com HP, em crise ou contínua, foram mantidos em ventilação mecânica, hiperventilação moderada e curarizados até uma relação PPS/PAS $<40 \%$. Nos casos em que não houve queda significante da pressão pulmonar com uso de drogas vasodilatadoras pulmonares, sedação e hiperventilação pulmonar, foi introduzido óxido nítrico no circuito da ventilação pulmonar.

\section{f) Metodologia Estatística}

Para comparar os grupos UFC e UFC+UFM com relação às variáveis de homogeneidade e eficácia, os seguintes métodos estatísticos foram empregados:
Teste Qui-quadrado de Pearson ou Teste exato de Fisher: empregados para avaliar comparativamente os 2 Grupos quanto à homogeneidade de proporções. O segundo teste foi considerado somente para tabelas de contingência que apresentavam valores esperados inferiores a 5 .

Teste t-Student ou teste da soma de postos de Wilcoxon: empregados para avaliar comparativamente os Grupos quanto aos dados quantitativos. $O$ primeiro método permite a comparação entre as médias dos 2 Grupos. Somente quando as suposições necessárias para o uso deste método não foram verificadas, utilizou-se o teste não-paramétrico de Wilcoxon, que permite a comparação das medianas dos 2 grupos.

Método de Kaplan-Meier e teste de "log-rank": utilizado para estimar a probabilidade de alta hospitalar. O teste de "log-rank" foi empregado para comparar as curvas de Kaplan-Meier estimadas para cada grupo.

\section{RESULTADOS}

Resultados da Comparação Entre os Grupos com Relação às Variáveis de Homogeneidade

Com relação aos resultados das variáveis de homogeneidade apresentados na Tabela 1, observou-se que não houve diferença estatisticamente significante entre os Grupos UFC e UFC+UFM, quanto aos valores médios das variáveis:

- Razão peso-idade

- Tempo de anóxia

- Tempo de CEC

Observou-se que a média de idades e o peso médio é maior no Grupo UFC; no entanto, não foi detectada diferença estatística entre os Grupos.

Com relação às variáveis da Tabela 2 (sexo, presença de hipertensão pulmonar, presença de cianose e grau de desnutrição), os grupos UFC e UFC+UFM são homogêneos. Quanto à complexidade do risco cirúrgico, existe um número maior de casos com complexidade 3 ou 4 no Grupo UFC+UFM; entretanto, não foi detectada diferença estatisticamente significante com relação ao Grupo UFC.

Resultados da Comparação Entre os Grupos com Relação às Variáveis de Eficácia

A partir dos resultados da Tabela 3, observouse que: 
Maluf MA, Mangia C, Bertuccez J, Silva C, Catani R, Carvalho W, Carvalho A, Buffolo E - Estudo comparativo da ultrafiltração convencional e associação de ultrafiltração convencional e modificada na correção de cardiopatias congênitas com alto risco cirúrgico. Rev Bras Cir Cardiovasc 1999; 14 (3): 221-36

TABELA 1

\begin{tabular}{|c|c|c|c|c|c|c|c|c|}
\hline VARIÁVEL & GRUPO & No & MíNIMO & MÁXIMO & MEDIANA & MÉDIA & $\begin{array}{c}\text { DESVIO } \\
\text { PADRÃOO }\end{array}$ & $P$ \\
\hline Idade & $\begin{array}{l}\text { UFC } \\
\text { UFC + UFM }\end{array}$ & $\begin{array}{l}21 \\
20\end{array}$ & $\begin{array}{l}0,50 \\
0,30\end{array}$ & $\begin{array}{l}36,00 \\
36,00\end{array}$ & $\begin{array}{r}10,00 \\
5,50\end{array}$ & $\begin{array}{r}15,07 \\
9,21\end{array}$ & $\begin{array}{r}11,87 \\
8,51\end{array}$ & $0,085^{\#}$ \\
\hline Peso & $\begin{array}{l}\text { UFC } \\
\text { UFC + UFM }\end{array}$ & $\begin{array}{l}21 \\
20\end{array}$ & $\begin{array}{l}3,60 \\
2,20\end{array}$ & $\begin{array}{l}13,50 \\
12,00\end{array}$ & $\begin{array}{l}6,65 \\
5,40\end{array}$ & $\begin{array}{l}7,27 \\
5,77\end{array}$ & $\begin{array}{l}3,07 \\
2,54\end{array}$ & $0,096^{*}$ \\
\hline Razão peso-idade & $\begin{array}{l}\text { UFC } \\
\text { UFC + UFM }\end{array}$ & $\begin{array}{l}21 \\
20\end{array}$ & $\begin{array}{l}0,51 \\
0,45\end{array}$ & $\begin{array}{l}1,00 \\
0,91\end{array}$ & $\begin{array}{l}0,71 \\
0,72\end{array}$ & $\begin{array}{l}0,72 \\
0,71\end{array}$ & $\begin{array}{l}0,14 \\
0,13\end{array}$ & $0,669^{*}$ \\
\hline Tempo de anóxia & $\begin{array}{l}\text { UFC } \\
\text { UFC + UFM }\end{array}$ & $\begin{array}{l}21 \\
20\end{array}$ & $\begin{array}{r}16,00 \\
0,00\end{array}$ & $\begin{array}{r}87,00 \\
102,00\end{array}$ & $\begin{array}{l}49,00 \\
56,50\end{array}$ & $\begin{array}{l}51,00 \\
52,20\end{array}$ & $\begin{array}{l}22,46 \\
27,68\end{array}$ & 0,879 \\
\hline Tempo de CEC & $\begin{array}{l}\text { UFC } \\
\text { UFC + UFM }\end{array}$ & $\begin{array}{l}21 \\
20\end{array}$ & $\begin{array}{l}45,00 \\
50,00\end{array}$ & $\begin{array}{l}300,00 \\
191,00\end{array}$ & $\begin{array}{l}100,00 \\
103,00\end{array}$ & $\begin{array}{l}101,52 \\
109,30\end{array}$ & $\begin{array}{l}54,76 \\
44,13\end{array}$ & $0,620^{*}$ \\
\hline
\end{tabular}

\#: teste de Wilcoxon

*: teste t-Student para 2 amostras independentes

TABELA 2

DISTRIBUIÇÃO DE FREQÜÊNCIA E COMPARAÇÃO ENTRE OS GRUPOS COM RELAÇÃO ÀS VARIÁVEIS DE HOMOGENEIDADE

\begin{tabular}{|c|c|c|c|c|c|c|c|}
\hline \multirow{3}{*}{ VARIÁVEL } & \multirow{3}{*}{ CATEGORIA } & \multirow{2}{*}{\multicolumn{2}{|c|}{ UFC }} & \multicolumn{2}{|c|}{ GRUPO } & \multirow{3}{*}{ TOTAL } & \multirow{3}{*}{$P$} \\
\hline & & & & & $F M$ & & \\
\hline & & No & $\%$ & No & $\%$ & & \\
\hline Sexo & $\begin{array}{l}\text { Feminino } \\
\text { Masculino }\end{array}$ & $\begin{array}{l}11 \\
10\end{array}$ & $\begin{array}{l}52,4 \\
47,6\end{array}$ & $\begin{array}{l}10 \\
10\end{array}$ & $\begin{array}{l}50,0 \\
50,0\end{array}$ & $\begin{array}{l}21 \\
20\end{array}$ & 0,879 \\
\hline Hipertensão pulmonar & $\begin{array}{l}\text { Não } \\
\text { Sim }\end{array}$ & $\begin{array}{c}6 \\
15\end{array}$ & $\begin{array}{l}28,6 \\
71,4\end{array}$ & $\begin{array}{c}6 \\
14\end{array}$ & $\begin{array}{l}30,0 \\
70,0\end{array}$ & $\begin{array}{l}12 \\
29\end{array}$ & 0,920 * \\
\hline Cianose & $\begin{array}{l}\text { Não } \\
\text { Sim }\end{array}$ & $\begin{array}{c}14 \\
7\end{array}$ & $\begin{array}{l}66,6 \\
33,3\end{array}$ & $\begin{array}{c}11 \\
9\end{array}$ & $\begin{array}{l}55,0 \\
45,0\end{array}$ & $\begin{array}{l}25 \\
16\end{array}$ & $0,444^{*}$ \\
\hline Nutrição & $\begin{array}{l}\text { Normal } \\
\text { Leve }\end{array}$ & $\begin{array}{l}4 \\
4\end{array}$ & $\begin{array}{l}19,1 \\
19,1\end{array}$ & $\begin{array}{l}1 \\
9\end{array}$ & $\begin{array}{l}5,0 \\
45,0\end{array}$ & $\begin{array}{c}5 \\
13\end{array}$ & $0,233^{*}$ \\
\hline Desnutrição & $\begin{array}{l}\text { Moderada } \\
\text { Grave }\end{array}$ & $\begin{array}{l}8 \\
5\end{array}$ & $\begin{array}{l}38,0 \\
23,8\end{array}$ & $\begin{array}{l}5 \\
5\end{array}$ & $\begin{array}{l}25,0 \\
25,0\end{array}$ & $\begin{array}{l}13 \\
10\end{array}$ & \\
\hline Grau de Complexidade & 2 & 16 & 76,2 & 10 & 50,0 & 26 & $0,082^{*}$ \\
\hline Cirúrgica & 3 ou 4 & 5 & 23,8 & 10 & 50,0 & 15 & \\
\hline
\end{tabular}

Não houve diferença estatisticamente significante entre os volumes médios obtidos por meio da UFC nos dois Grupos; entretanto, o volume total é significativamente maior no Grupo UFC+UFM. Além disso, a relação entre o volume total extraído na ultrafiltração e o volume do perfusato é significativamente maior no grupo UFC+UFM.

Com relação ao tempo total de uso de drogas, tempo de intubação, tempo de UTIP e tempo de permanência hospitalar, não se identificou diferença estatisticamente significante entre os Grupos UFC+UFM e UFC.

As Tabelas 4 e 5 mostram a evolução pósoperatória dos pacientes. No Grupo UFC houve seis óbitos. Destes, 5 (83,3\%) apresentavam HP e no
Grupo UFC+UFM, os 4 (100\%) que faleceram tinham HP.

Com relação ao tempo de permanência hospitalar não houve diferença significante entre os grupos, analisados em cinco tempos diferentes (Tabela 6 e Figura 2).

\section{COMENTÁRIOS}

Este trabalho consiste de um estudo piloto envolvendo pacientes submetidos à correção cirúrgica de cardiopatias congênitas de moderada a grave complexidade. 
Maluf MA, Mangia C, Bertuccez J, Silva C, Catani R, Carvalho W, Carvalho A, Buffolo E - Estudo comparativo da ultrafiltração convencional e associação de ultrafiltração convencional e modificada na correção de cardiopatias congênitas com alto risco cirúrgico. Rev Bras Cir Cardiovasc 1999; 14 (3): 221-36

TABELA 3

MEDIDAS DESCRITIVAS E COMPARAÇÃO ENTRE OS GRUPOS COM RELAÇÃO ÀS VARIÁVEIS DE EFICÁCIA

\begin{tabular}{|c|c|c|c|c|c|c|c|c|}
\hline VARIÁVEL & & № & MÍNIMO & MÁXIMO & MEDIANA & MÉDIA & $\begin{array}{l}\text { DESVIO } \\
\text { PADRÃOO }\end{array}$ & $\boldsymbol{P}$ \\
\hline $\begin{array}{l}\text { Volume UFC } \\
\text { (ml) }\end{array}$ & $\begin{array}{c}\text { UFC } \\
\text { UFC+UFM }\end{array}$ & $\begin{array}{l}21 \\
20\end{array}$ & $\begin{array}{l}50,00 \\
65,00\end{array}$ & $\begin{array}{l}250,00 \\
270,00\end{array}$ & $\begin{array}{l}130,00 \\
125,00\end{array}$ & $\begin{array}{l}143,33 \\
130,75\end{array}$ & $\begin{array}{l}54,34 \\
55,45\end{array}$ & $0,467^{*}$ \\
\hline Volume UFM (ml) & UFC + UFM & 20 & 50,00 & 150,00 & 100,00 & 96,25 & 30,47 & \\
\hline $\begin{array}{l}\text { Volume total de } \\
\text { ultrafiltração (VTU) (ml) }\end{array}$ & $\begin{array}{c}\text { UFC } \\
\text { UFC + UFM }\end{array}$ & $\begin{array}{l}21 \\
20\end{array}$ & $\begin{array}{c}50,00 \\
125,00\end{array}$ & $\begin{array}{l}250,00 \\
360,00\end{array}$ & $\begin{array}{l}130,00 \\
235,00\end{array}$ & $\begin{array}{l}143,33 \\
227,00\end{array}$ & 71,44 & $<0,001^{*}$ \\
\hline $\begin{array}{l}\text { Volemia do paciente } \\
(\mathrm{ml})\end{array}$ & $\begin{array}{c}\text { UFC } \\
\text { UFC + UFM }\end{array}$ & $\begin{array}{l}21 \\
20\end{array}$ & $\begin{array}{l}288,00 \\
176,00\end{array}$ & $\begin{array}{c}1080,00 \\
960,00\end{array}$ & $\begin{array}{l}532,00 \\
432,00\end{array}$ & $\begin{array}{l}581,90 \\
461,60\end{array}$ & $\begin{array}{l}245,80 \\
203,20\end{array}$ & ---- \\
\hline $\begin{array}{l}\text { Volume do perfusato } \\
\text { (V. perfusato) (ml) }\end{array}$ & $\begin{array}{c}\text { UFC } \\
\text { UFC + UFM }\end{array}$ & $\begin{array}{l}21 \\
20\end{array}$ & $\begin{array}{l}738,00 \\
626,00\end{array}$ & $\begin{array}{l}1530,00 \\
1410,00\end{array}$ & $\begin{array}{l}982,00 \\
882,00\end{array}$ & $\begin{array}{c}1031,43 \\
911,60\end{array}$ & $\begin{array}{l}245,75 \\
203,20\end{array}$ & ---- \\
\hline $\begin{array}{l}\text { Relação entre VTU e } \\
\text { V. perfusato }\end{array}$ & $\begin{array}{c}\text { UFC } \\
\text { UFC + UFM }\end{array}$ & $\begin{array}{l}21 \\
20\end{array}$ & $\begin{array}{c}6,78 \\
12,06\end{array}$ & $\begin{array}{l}24,69 \\
49,32\end{array}$ & $\begin{array}{l}14,35 \\
27,32\end{array}$ & $\begin{array}{l}14,16 \\
26,04\end{array}$ & $\begin{array}{l}5,16 \\
9,91\end{array}$ & $<0,001^{*}$ \\
\hline $\begin{array}{l}\text { Tempo total de uso de } \\
\operatorname{drogas}^{* *}(\mathrm{~h})\end{array}$ & $\begin{array}{c}\text { UFC } \\
\text { UFC + UFM }\end{array}$ & $\begin{array}{l}15 \\
15\end{array}$ & $\begin{array}{l}42,00 \\
12,00\end{array}$ & $\begin{array}{l}288,00 \\
528,00\end{array}$ & $\begin{array}{c}144,00 \\
96,00\end{array}$ & $\begin{array}{l}145,20 \\
137,60\end{array}$ & $\begin{array}{c}89,58 \\
132,77\end{array}$ & $0,855^{*}$ \\
\hline Tempo de intubação** $(\mathrm{h})$ & $\begin{array}{c}\text { UFC } \\
\text { UFC + UFM }\end{array}$ & $\begin{array}{l}15 \\
15\end{array}$ & $\begin{array}{l}24,00 \\
12,00\end{array}$ & $\begin{array}{l}192,00 \\
288,00\end{array}$ & $\begin{array}{l}78,00 \\
72,00\end{array}$ & $\begin{array}{l}94,80 \\
95,67\end{array}$ & $\begin{array}{l}66,20 \\
90,29\end{array}$ & $0,976^{*}$ \\
\hline $\begin{array}{l}\text { Tempo de UTIP** } \\
\text { (h) }\end{array}$ & $\begin{array}{c}\text { UFC } \\
\text { UFC + UFM }\end{array}$ & $\begin{array}{l}15 \\
16\end{array}$ & $\begin{array}{l}66,00 \\
24,00\end{array}$ & $\begin{array}{l}312,00 \\
576,00\end{array}$ & $\begin{array}{l}144,00 \\
120,50\end{array}$ & $\begin{array}{l}169,60 \\
157,81\end{array}$ & $\begin{array}{c}90,61 \\
150,80\end{array}$ & $0,795^{*}$ \\
\hline $\begin{array}{l}\text { Tempo de permanência } \\
\text { hospitalar }\end{array}$ & $\begin{array}{c}\text { UFC } \\
\text { UFC + UFM }\end{array}$ & $\begin{array}{l}15 \\
16\end{array}$ & $\begin{array}{l}5,00 \\
4,00\end{array}$ & $\begin{array}{l}28,00 \\
43,00\end{array}$ & $\begin{array}{l}15,00 \\
12,00\end{array}$ & $\begin{array}{l}14,87 \\
14,69\end{array}$ & $\begin{array}{l}5,57 \\
9,67\end{array}$ & $0,950^{*}$ \\
\hline $\begin{array}{l}\text { Número de horas de diálise } \\
\text { peritoneal }\end{array}$ & $\begin{array}{c}\text { UFC } \\
\text { UFC + UFM }\end{array}$ & $\begin{array}{l}5 \\
5\end{array}$ & $\begin{array}{c}8,00 \\
24,00\end{array}$ & $\begin{array}{l}192,00 \\
144,00\end{array}$ & $\begin{array}{l}48,00 \\
48,00\end{array}$ & $\begin{array}{l}81,20 \\
69,60\end{array}$ & $\begin{array}{l}82,46 \\
49,77\end{array}$ & ---- \\
\hline
\end{tabular}

${ }^{*}$ : teste $\mathrm{t}$-Student para 2 amostras independentes

**: Medidas descritivas desconsiderando os óbitos

Trata-se, portanto, de um estudo comparativo, prospectivo e consecutivo, no-randomizado, semeIhante a outras publicações (12-16).

A randomização dos pacientes, sem dúvida constitui uma situação ideal, porque permite uma acurada avaliação dos resultados $(7,17)$. No entanto, trabaIhos no-comparativos(18) têm restrições na avaliação dos benefícios do uso da UFM.

O interesse pelo emprego da técnica da UFM vem crescendo e justificando a realização de estudos multicentricos nos EUA, como o demonstraram DARLING et al. (19). Participaram deste estudo 50 centros de cirurgia cardíaca infantil, com um índice de aprovação da UFM em torno de 44\% (22 centros). Este trabalho cooperativo teve como objetivo avaliar a técnica e os resultados.

Apesar da experiência adquirida, foram detectadas complicações técnicas em $82 \%$ destas Instituições, sendo a mais freqüente a entrada de ar no circuito $(68,1 \%)$.

O desenho da nossa pesquisa ofereceu oportunidade de ultrafiltração a ambos os Grupos, cujo perfil de idade, peso e complexidade cirúrgica definiramnos como de alto risco. Todos os pacientes receberam a UFC, permitindo, assim, a ultrafiltração de mediadores de resposta inflamatória liberados princi- palmente na fase de aquecimento da CEC (20). Não encontramos, porém, trabalhos semelhantes ao nosso, onde o grupo em estudo foi submetido à associação de UFC e UFM.

Originalmente, este trabalho foi constituído por pacientes de 0 a 14 anos de idade. Em uma avaliação prévia foi possível concluir que pacientes com idade acima de 36 meses, apesar de constituir um grupo heterogêneo, não apresentavam diferenças, quando comparados os Grupos com UFC e UFC + UFM. Estes achados, em um Grupo de pacientes pouco homogêneo e a necessidade de avaliar os resultados em neonatos e lactentes, com alto risco cirúrgico, nos motivou a definir nossa pesquisa em pacientes com idade de 0 a 36 meses. Pacientes com faixa etária semelhante formaram parte de publicações recentes $(14,17,18,21)$.

A inclusão no trabalho de 41 pacientes operados que tiveram oportunidade de participar do estudo e serem acompanhados durante sua evolução pós-operatória, embora não seja um número expressivo, permitiu uma análise detalhada de variáveis e obtenção de conclusões relevantes, semelhante a outros trabalhos publicados $(7,13,15,16,18,22)$

Apesar de nossos grupos de UFC e UFC+UFM serem homogêneos e suas idades não apresenta- 
Maluf MA, Mangia C, Bertuccez J, Silva C, Catani R, Carvalho W, Carvalho A, Buffolo E - Estudo comparativo da ultrafiltração convencional e associação de ultrafiltração convencional e modificada na correção de cardiopatias congênitas com alto risco cirúrgico. Rev Bras Cir Cardiovasc 1999; 14 (3): 221-36

TABELA 4

\begin{tabular}{|c|c|c|c|c|c|c|c|c|}
\hline \multicolumn{9}{|c|}{$\begin{array}{l}\text { EVOLUÇÃO PÓS-OPERATÓRIA IMEDIATA } \\
\text { GRUPO I-ULTRAFILTRAÇÃO CONVENCIONAL }\end{array}$} \\
\hline № & DIAGNÓSTICO & & $\begin{array}{l}\text { S } \\
\text { VAS } \\
\text { (h) }\end{array}$ & $\begin{array}{c}\text { TEMPO } \\
\text { INTUB. } \\
\text { (h) }\end{array}$ & $\begin{array}{l}\text { UTIP } \\
\text { (h) }\end{array}$ & $\begin{array}{l}\text { DIÁLISE } \\
\text { (h) }\end{array}$ & $\begin{array}{l}\text { INT. HOSP. } \\
\text { (DIAS) }\end{array}$ & EVOLUÇÃO \\
\hline 1 & $\mathrm{TGA}+\mathrm{CIA}$ & 2 & 264 & 120 & 264 & 14 & 28 & Boa \\
\hline 2 & $\mathrm{CIV}+\mathrm{HP}$ & 2 & 48 & 48 & 48 & -- & -- & Óbito \\
\hline 3 & $D S A V-T+I M+H P$ & 1 & 48 & 24 & 72 & -- & 15 & Boa \\
\hline 4 & DSAV-T + PCA + HP & 2 & 144 & 96 & 144 & -- & 13 & Boa \\
\hline 5 & DSAV-T + HP & 3 & 48 & 48 & 48 & 8 & -- & Óbito \\
\hline 6 & $\begin{array}{l}\text { DVSVD + CIV SUB PUL } \\
+\mathrm{HP}\end{array}$ & 2 & 9 & 9 & 9 & - & - & Óbito \\
\hline 7 & $\mathrm{TGA}+\mathrm{CIA}$ & 3 & 288 & 192 & 312 & 192 & 23 & Boa \\
\hline 8 & Tétrade de Fallot & 3 & 216 & 120 & 240 & 144 & 15 & Boa \\
\hline 9 & DATVP + HP & 2 & 24 & 24 & 24 & -- & -- & Óbito \\
\hline 10 & $\mathrm{CIV}+\mathrm{HP}$ & 1 & 42 & 24 & 66 & - & 8 & Boa \\
\hline 11 & $\begin{array}{l}\text { DSAV-T + HP + S } \\
\text { Down }\end{array}$ & 1 & 72 & 78 & 102 & - & 17 & Boa \\
\hline 12 & $\mathrm{CIV}+\mathrm{PCA}+\mathrm{HP}$ & 1 & 96 & 72 & 96 & -- & 11 & Boa \\
\hline 13 & $\mathrm{CIV}+\mathrm{HP}$ & 2 & 240 & 192 & 240 & - & 15 & Boa \\
\hline 14 & $\mathrm{CIV}+\mathrm{CIA}+\mathrm{PCA}+\mathrm{HP}$ & 2 & 240 & 192 & 264 & - & 15 & Boa \\
\hline 15 & Tétrade de Fallot & 2 & 192 & 24 & 192 & - & 16 & Boa \\
\hline 16 & $\mathrm{TGA}+\mathrm{CIA}$ & 3 & 48 & 72 & 120 & 48 & 12 & Boa \\
\hline 17 & $\mathrm{CIV}+\mathrm{IM}+\mathrm{HP}$ & 1 & 72 & 24 & 72 & - & 5 & Boa \\
\hline 18 & $\mathrm{ClA}+\mathrm{PCA}+\mathrm{HP}$ & 3 & 48 & 24 & 72 & - & 12 & Boa \\
\hline 19 & $\mathrm{CIV}+\mathrm{PCA}+\mathrm{HP}$ & 1 & 168 & 168 & 288 & - & 18 & Boa \\
\hline 20 & $\mathrm{CIV}+\mathrm{PCA}+\mathrm{HP}$ & 3 & 8 & 8 & 8 & - & -- & Óbito \\
\hline 21 & Origem anômala de ACE & 3 & 6 & 6 & 6 & - & - & Óbito \\
\hline
\end{tabular}

rem diferenças estatisticamente significantes $(\mathrm{p}=$ 0,085), o Grupo UFC+UFM apresentava idade mínima e média inferior ao do Grupo UFC: 0,30 vs. 0,50 meses e 9,21 $\pm 8,51$ vs. $15,07 \pm 11,87$ meses, respectivamente.

A faixa etária dos pacientes nos trabalhos publicados sobre o assunto foi superior quando comparada ao nosso grupo. Na pesquisa de NAIK et al. (7), a média da idade foi 48 meses; no trabalho de JOURNOIS et al. ${ }^{(17)}$, em 1996, foi 49 meses, no Grupo controle e 1,10 anos, no Grupo de UFM; na pesquisa de DRAAISMA et al. (13) no Grupo de UFC, foi 33,6 meses e no Grupo de UFM, 31,8 meses; no trabalho de $A D$ et al. (22) o Grupo controle tinha 35 meses em média e o Grupo de UFM, 24,9 meses. Apenas no trabalho de DAVIES et al. (21) os pacientes tinham média de idade inferior ao nosso grupo: 8 meses no Grupo controle e 6 meses no Grupo de UFM.

A classificação dos pacientes em grau de complexidade cirúrgica utilizada nesta pesquisa foi baseada no trabalho de JENKINS et al (10), que examinaram o impacto da mortalidade hospitalar em 2833 pacientes submetidos à correção cirúrgica de cardiopatias congênitas, em 37 centros da Califórnia e hospital de Massachusetts, em 1988 e 1989, respectivamente. Quatro categorias foram definidas segundo o código principal ou secundário da "International Classification of Diseases, Nineth Revision Clinical Modification" (ICD-9-CM).

Apesar da análise detalhada de trabalhos que dizem respeito ao tema de ultrafiltração, não encontramos referência a formas de classificação dos pacientes, conforme seu grau de complexidade.

No nosso trabalho, apesar de não haver diferença estatisticamente significante entre os Grupos UFC+UFM e UFC $(p=0,150)$, havia $50 \%$ de pacientes do Grupo UFC+UFM na categoria 3 e 4 (mais grave), ao passo que no Grupo UFC, $76,2 \%$ dos casos pertenciam a categoria 2 (menos grave).

Analisando os trabalhos publicados sobre o assunto e utilizados os mesmos critérios de classificação de JENKINS et al. (10), observamos que na casuística de NAIK et al. (7) apenas $16 \%$ dos pacientes pertenciam à categoria 3 e 4, no Grupo UFM e $28 \%$ dos pacientes a esta mesma categoria, no Grupo controle.

No trabalho de DAVIES et al. (21) apenas $27 \%$ 
Maluf MA, Mangia C, Bertuccez J, Silva C, Catani R, Carvalho W, Carvalho A, Buffolo E - Estudo comparativo da ultrafiltração convencional e associação de ultrafiltração convencional e modificada na correção de cardiopatias congênitas com alto risco cirúrgico. Rev Bras Cir Cardiovasc 1999; 14 (3): 221-36

TABELA 5

\begin{tabular}{|c|c|c|c|c|c|c|c|c|}
\hline \multirow[b]{2}{*}{ No } & \multicolumn{8}{|c|}{$\begin{array}{c}\text { EVOLUČÃO PÓS-OPERATÓRIA IMEDIATA } \\
\text { GRUPO II- ULTRAFILTRAÇÃ́O CONVENCIONAL + ULTRAFILTRAÇÃO MODIFICADA }\end{array}$} \\
\hline & DIAGNÓSTICO & & $\begin{array}{l}S \\
\text { VAS } \\
(h)\end{array}$ & $\begin{array}{c}\text { TEMPO } \\
\text { INTUB. } \\
\text { (h) }\end{array}$ & $\begin{array}{l}\text { UTIP } \\
\text { (h) }\end{array}$ & $\begin{array}{l}\text { DIÁLISE } \\
\text { (h) }\end{array}$ & $\begin{array}{l}\text { INT. HOSP. } \\
\text { (DIAS) }\end{array}$ & EVOLUÇÃO \\
\hline 1 & DSAV-T + HP & 1 & 72 & 36 & 48 & - & 10 & Boa \\
\hline 2 & $\mathrm{CIV}+\mathrm{HP}$ & 1 & 48 & 12 & 48 & -- & 10 & Boa \\
\hline 3 & $\mathrm{CIV}+\mathrm{HP}$ & 1 & 48 & 24 & 36 & - & 8 & Boa \\
\hline 4 & $\begin{array}{l}\text { DVSVD + CIV } \\
\text { sub-pul + HP }\end{array}$ & 1 & 120 & 60 & 120 & 24 & 14 & Boa \\
\hline 5 & $\begin{array}{l}\text { TGA + CIV } \\
\text { Band. Pul. }\end{array}$ & 3 & 144 & 120 & 168 & 48 & 14 & Boa \\
\hline 6 & $\begin{array}{l}\text { Cor } \\
\text { triatriatum + HP }\end{array}$ & 3 & 48 & 48 & 48 & 36 & -- & Óbito \\
\hline 7 & $\mathrm{TGA}+\mathrm{ClA}+\mathrm{HP}$ & 3 & 48 & 48 & 48 & - & - & Óbito \\
\hline 8 & civ $+h p$ & 1 & 12 & 12 & 24 & - & 9 & Boa \\
\hline 9 & $\begin{array}{l}\text { DSAV-T } \\
+\mathrm{IM}+\mathrm{HP}\end{array}$ & 1 & 96 & 72 & 144 & - & 14 & Boa \\
\hline 10 & $\begin{array}{l}\text { Truncus } \\
\text { arteriosus tipo I }\end{array}$ & 2 & 72 & 72 & 72 & -- & - & Óbito \\
\hline 11 & $\begin{array}{l}\text { DSAV-T } \\
+\mathrm{HP}+\mathrm{S} \text {. Down }\end{array}$ & 2 & 48 & 216 & 216 & -- & -- & Óbito \\
\hline 12 & $\begin{array}{l}\mathrm{CIV}+\mathrm{PCA}+\mathrm{HP}+ \\
\text { S. Down }\end{array}$ & 2 & 168 & 96 & 240 & - & 43 & Boa \\
\hline 13 & $\mathrm{CIV}+\mathrm{HP}$ & 1 & 288 & 264 & 312 & -- & 16 & Boa \\
\hline 14 & $\begin{array}{l}\text { Est. APD- } \\
\text { APE + PCA }\end{array}$ & 1 & 12 & 12 & 48 & -- & 8 & Boa \\
\hline 15 & $\mathrm{CIV}+\mathrm{CIA}+\mathrm{HP}$ & 2 & 96 & 96 & 120 & - & 10 & Boa \\
\hline 16 & $\mathrm{CIV}+\mathrm{HP}$ & 0 & 0 & 0 & 24 & -- & 4 & Boa \\
\hline 17 & $\begin{array}{l}\text { DVEVD+EPV+ } \\
\text { TGA }\end{array}$ & 2 & 72 & 55 & 89 & - & 10 & Boa \\
\hline 18 & $\mathrm{TGA}+\mathrm{CIA}$ & 2 & 120 & 72 & 168 & - & 14 & Boa \\
\hline 19 & $T G A+C I A$ & 3 & 528 & 288 & 576 & 96 & 30 & Boa \\
\hline 20 & $T G A+C I A$ & 4 & 240 & 216 & 360 & 144 & 21 & Boa \\
\hline
\end{tabular}

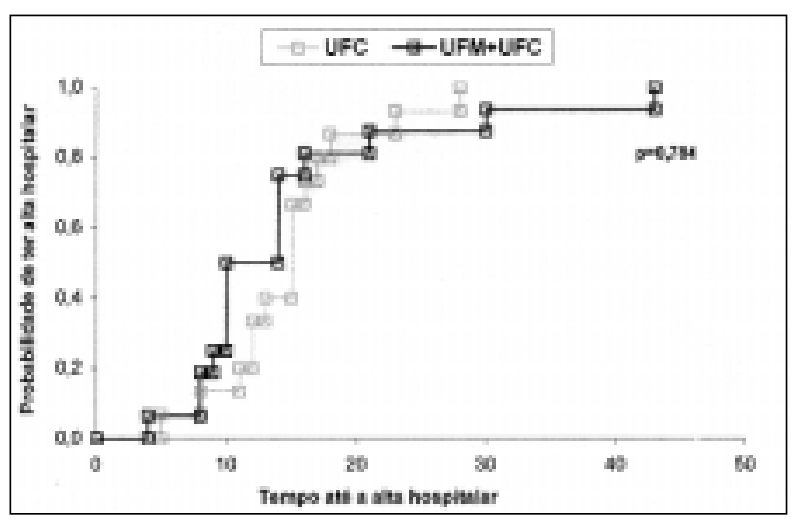

Fig. 2 - Tempo de alta hospitalar efetivo em relação a probabilidade calculada.

dos pacientes estava na categoria 3 e 4, no Grupo UFM e $30 \%$ dos casos, nesta categoria, no Grupo controle.
No trabalho de JOURNOIS et al. (17) somente $30 \%$ dos pacientes formaram parte da categoria 3 e 4, no grupo UFM e $40 \%$ dos casos, pertenciam a esta mesma categoria, no Grupo controle.

A associação de cardiopatia congênita e hipertensão pulmonar moderada a grave (relação pressão pulmonar/pressão sistêmica $\geq 60 \%$ ) representa um grupo de maior risco cirúrgico e necessita de tratamento efetivo para controle da HP. Nestes casos, estimam-se tempos maiores de intubação e permanência em UTIP.

No nosso trabalho, $70 \%$ dos pacientes do grupo UFC+UFM e $71,4 \%$ dos casos do grupo UFC apresentavam HP, com moderada à grave repercussão hemodinâmica.

No trabalho de NAIK et al. (7) a HP estava presente apenas em $32 \%$ dos pacientes, do grupo UFM e $44 \%$ dos casos, do grupo controle. 
Maluf MA, Mangia C, Bertuccez J, Silva C, Catani R, Carvalho W, Carvalho A, Buffolo E - Estudo comparativo da ultrafiltração convencional e associação de ultrafiltração convencional e modificada na correção de cardiopatias congênitas com alto risco cirúrgico. Rev Bras Cir Cardiovasc 1999; 14 (3): 221-36

TABELA 6

\begin{tabular}{|c|c|c|c|c|c|c|}
\hline GRUPO & $\begin{array}{c}N o D E \\
\text { PACIENTES } \\
\text { SOBREVIVENTES }\end{array}$ & $\begin{array}{c}\text { No }{ }^{\circ} \text { DE } \\
\text { EVENTOS }\end{array}$ & $\begin{array}{c}\text { TEMPO } \\
\text { (DIAS) }\end{array}$ & $\begin{array}{c}\text { PROBABILIDADE } \\
\text { DE TER ALTA } \\
\text { HOSPITALAR }\end{array}$ & $\begin{array}{c}E R R O \\
P A D R \tilde{A} O\end{array}$ & $\begin{array}{c}\text { NoDE } \\
\text { INDIVÍDUOS } \\
\text { EM RISCO }\end{array}$ \\
\hline UFC & 15 & 15 & $\begin{array}{c}5 \\
10 \\
15 \\
20 \\
30\end{array}$ & $\begin{array}{l}0,067 \\
0,133 \\
0,667 \\
0,867 \\
1,000\end{array}$ & $\begin{array}{l}0,064 \\
0,088 \\
0,122 \\
0,088 \\
0,000\end{array}$ & $\begin{array}{l}14 \\
13 \\
5 \\
2 \\
0\end{array}$ \\
\hline UFC + UFM & 16 & 16 & $\begin{array}{c}5 \\
10 \\
15 \\
20 \\
30\end{array}$ & $\begin{array}{l}0,063 \\
0,500 \\
0,750 \\
0,833 \\
0,938\end{array}$ & $\begin{array}{l}0,060 \\
0,125 \\
0,108 \\
0,098 \\
0,060\end{array}$ & $\begin{array}{c}15 \\
8 \\
4 \\
3 \\
1\end{array}$ \\
\hline
\end{tabular}

A casuística de JOURNOIS et al. (17) apresentava HP em $60 \%$ dos casos, do grupo UFM e $60 \%$ dos casos, do grupo UFC.

No estudo de DAVIES et al. (21) havia $63,6 \%$ de casos, no grupo UFM e $50 \%$ de casos, no grupo UFC, com HP.

A CEC é, sem dúvida, a responsável pelo aumento do conteúdo de água corporal provavelmente como resposta a uma reação inflamatória generalizada (23-25).

O aumento do conteúdo de água corporal é mais expressivo em crianças de baixo peso, submetidas a tempos prolongados de CEC e baixas temperaturas. $O$ excesso de água corporal pode provocar uma significativa disfunção orgânica, particularmente do pulmão, coração e cérebro.

No nosso trabalho, não houve diferença significativa entre os tempos de CEC que variaram entre 50 e 191 min. $(M=109,30 \pm 44,13)$ no grupo UFC + UFM e entre 45 e 300min. $(M=101,52 \pm 54,76)$, no grupo UFC $(p=0,620)$, utilizando hipotermia de 25 a $28^{\circ} \mathrm{C}$

No trabalho de NAIK et al. ${ }^{(8)}$ os tempos de CEC, no grupo UFM, variaram entre 24 e $190 \mathrm{~min}$ $(\mathrm{M}=97,92)$ e no grupo controle, de 11 a $178 \mathrm{~min}$. $(\mathrm{M}=75,68)$, utilizando hipotermia entre 17 e $28^{\circ} \mathrm{C}$.

$\mathrm{Na}$ casuística de DAVIES et al. (21) os tempos de CEC variaram entre 73 e $150 \mathrm{~min}$. $(M=106)$, no grupo UFM, enquanto no grupo de UFC, foi de 61 e $133(\mathrm{M}=92)$.

O emprego da ultrafiltração convencional em cirurgia cardíaca com auxílio da CEC foi iniciada a mais de 20 anos (ROMAGNOLI et al. (4) e DARUP ${ }^{(5)}$ ), constituindo-se num procedimento consagrado e reconhecido, que tem prestado importante contribuição no manejo do paciente no período per-operatório, permitindo eliminar o excesso de água corporal.
A necessidade de intervir em crianças com cardiopatias congênitas de maior complexidade, que requerem tempo prolongado de CEC, assim como as dificuldades no manejo do balanço hídrico no pós-operatório, nos motivou a iniciar o uso da UFC a partir de 1990, na Disciplina de Cirurgia Cardiovascular do Departamento de Cirurgia da EPMUNIFESP.

No entanto, tempos prolongados de CEC e baixas temperaturas, são necessários durante operações cardíacas em neonatos e lactentes, aumentando o risco de retenção de água corporal.

A evolução dos métodos que permitem retirar o excesso de líquido corporal, retido após CEC levou Elliot e seu grupo (NAIK et al. (8)) a introduzir modificações no sistema de ultrafiltração.

A UFM foi um assunto que despertou muito interesse em Congressos Internacionais de Cirurgia Cardíaca Pediátrica, nos últimos anos.

Inicialmente, o sistema da UFM utilizado foi semelhante ao descrito por NAIK et al. (8). No entanto, a necessidade de manter aquecido o sangue ultrafiltrado de retorno para o AD nos levou a introduzir mudanças na linha venosa (saída do hemoconcentrador), substituindo-a pela linha da cardioplegia e permitindo, assim, a passagem do sangue pelo permutador de calor, semelhante ao sistema descrito por GROOM et al. (12).

Um cuidadoso planejamento e preparo da técnica de UFM permitiu a nossos perfusionistas a execução do procedimento sem graves intercorrências.

No entanto, um paciente apresentou entrada de ar no circuito, por mecanismo de sucção em volta da cânula arterial, que foi resolvido com a diminuição da velocidade de ultrafiltração.

A entrada de ar no circuito, durante a UFM, acontece pela desproporção entre o diâmetro da 
Maluf MA, Mangia C, Bertuccez J, Silva C, Catani R, Carvalho W, Carvalho A, Buffolo E - Estudo comparativo da ultrafiltração convencional e associação de ultrafiltração convencional e modificada na correção de cardiopatias congênitas com alto risco cirúrgico. Rev Bras Cir Cardiovasc 1999; 14 (3): 221-36

aorta, às vezes hipoplásica, e a velocidade de ultrafiltração, ou pelo posicionamento da cânula aórtica, com seu orifício distal próximo à parede da aorta. A UFM, apesar de ser um procedimento simples e efetivo, não está isenta de complicações técnicas. Os cuidados na montagem do circuito, a cargo de um perfusionista experiente e sua execução, em sintonia com o cirurgião, poderão evitar acidentes.

O levantamento realizado por DARLING et al. (19) junto a perfusionistas de 22 centros, na Carolina do Norte (EUA), mostrou que complicações técnicas ocorreram em $82 \%$ destes centros, sendo a mais freqüente a entrada de ar no circuito $(68,1 \%)$.

Entre as desvantagens que são apontadas com a técnica de UFM, temos:

- A necessidade de manter as cânulas de Ao e AD durante o procedimento, provocando obstrução ao fluxo sangüíneo em pacientes com hipoplasia de Ao.

- A hipotensão arterial como conseqüência de drenagem excessiva pela linha arterial

- A queda da temperatura do sangue no circuito.

Estas intercorrências aconteceram na nossa experiência e foram contornadas utilizando cânulas apropriadas, reinfundindo no paciente o volume de sangue do reservatório venoso e aquecendo o sangue no permutador de calor da linha de cardioplegia, respectivamente.

A acumulação de água corpórea pode ser reconhecida clinicamente como a síndrome do "bebê de Michelin". A origem deste excesso de líquido ocorre pela administração de soluções por via intravenosa e pelo contato do perfusato com a volemia do paciente.

A relação volemia do paciente/perfusato, em crianças, é diferente dos adultos e se mantém sempre <1.

A CEC induz uma reação inflamatória generalizada, com desequilíbrio do meio interno do paciente, aumentando a retenção de água corporal. Estes efeitos têm maior impacto em crianças de baixo peso, que necessitam de tempo prolongado de CEC e baixo fluxo de perfusão, associados à hipotermia moderada ou profunda.

$\mathrm{Na}$ nossa Instituição, o uso da ultrafiltração durante a CEC (UFC) foi incorporado na rotina da correção cirúrgica das cardiopatias congênitas, em 1990.

Os benefícios que a UFC trouxe neste período foram inúmeros, dentre eles: redução do edema generalizado após correção cirúrgica e da necessidade de diálise peritoneal, estabilização hemodinâmica e ventilatória, redução das reoperações por sangramento e queda da mortalidade, mesmo em cardiopatias complexas.

Apesar da diminuição significante do perfusato, com a miniaturização dos oxigenadores e redução de diâmetros e comprimento das linhas do circuito extracorpóreo, o uso da UFC apresentou restrições relacionadas ao volume reduzido, especialmente em crianças pequenas.

Na evolução pós-operatória imediata, a reposição volêmica em crianças permite a melhora das condições hemodinâmicas, porém a presença de baixo débito urinário, provocará a retenção de líquido corporal, comprometendo a função cardíaca, pulmonar e cerebral. Nesta situação, uma resposta inadequada aos diuréticos obrigará a utilização de procedimentos como a diálise peritoneal ou ultrafiltração.

O emprego da diálise peritoneal permite a reversão do quadro de hipervolemia, porém, é um procedimento não totalmente livre de intercorrências.

Os métodos de ultrafiltração veno-venoso ou artério-venoso têm maior ou menor eficácia, dependendo do quadro hemodinâmico do paciente.

O volume de ultrafiltração, no grupo UFC + UFM, aumentou significativamente quando comparado a UFC isolada $(M=227 \pm 71,44 \mathrm{ml}$ vs $143,33 \pm 54,34 \mathrm{ml}$; $\mathrm{p}<0,001)$.

A UFM tem como principal vantagem ultrafiltrar apenas a volemia do paciente e permitir a reinfusão do sangue do circuito da CEC.

O uso da UFM, após a CEC permite a melhora da função sistólica do ventrículo esquerdo, em crianças submetidas à correção de defeitos congênitos, como demonstram os estudos de DAVIES et al. (21).

Os autores observaram aumento do diâmetro diastólico final e queda da pressão diastólica final do VE, após UFM, sendo interpretados estes dados como melhora da complacência ventricular, resultado de redução do edema miocárdico.

São vários os estudos que demonstram a melhora dos parâmetros hemodinâmicos, com elevação da pressão arterial, após UFM $(7,19,20,23-26)$. Os autores acreditam que há redução simultânea da resistência vascular pulmonar e conteúdo de água do miocárdio. GAINOR et al. (27) comprovaram a redução da área de secção transversa do miocárdio, após UFM.

Estudos realizados por ELLIOTT (28) demonstraram que o uso da UFM permitiu a queda da resistência vascular pulmonar, permanecendo inalterada a resistência vascular sistêmica.

A melhora hemodinâmica poderia estar relacionada à neutralização da resposta inflamatória ao uso de CEC, em razão da ultrafiltração dos mediadores proteicos ${ }^{(20,28,29)}$. Crianças submetidas a tempos prolongados de CEC, utilizando hipotermia e hemodiluição, poderão evoluir com importante edema 
Maluf MA, Mangia C, Bertuccez J, Silva C, Catani R, Carvalho W, Carvalho A, Buffolo E - Estudo comparativo da ultrafiltração convencional e associação de ultrafiltração convencional e modificada na correção de cardiopatias congênitas com alto risco cirúrgico. Rev Bras Cir Cardiovasc 1999; 14 (3): 221-36

miocárdico dificultando o fechamento do tórax, quando não forem adequadamente ultrafiltradas.

Em todos os 41 pacientes do nosso trabalho foi possível conduzí-los à UTIP com o esterno fechado. No entanto, 4 casos no trabalho de AD et al. ${ }^{(22)}$ e 3 casos no trabalho de DAVIES et al. (21) precisaram ser mantidos com o esterno aberto devido a importante edema miocárdico e instabilidade hemodinâmica.

Apesar da significativa melhora da função do VE com uso da UFM, demonstrado nos trabalhos de NAIK et al. (7) e DAVIES et al. (21), não houve diferença significante no tempo de uso de drogas inotrópicas, quando comparado ao Grupo controle.

$\mathrm{Na}$ nossa pesquisa, o tempo médio de uso de drogas inotrópicas foi semelhante em ambos os Grupos $(p=0,855)$.

A necessidade de tempos prolongados de uso de drogas inotrópicas poderia estar relacionada à piora da função do VE, decorrente da queda do $\mathrm{Ht}$ (sangramento), com comprometimento da liberação de oxigênio para os tecidos, ou relacionada ao tempo prolongado de CEC e anóxia miocárdica.

Os efeitos benéficos da UFM foram evidentes no trabalho de JOURNOIS et al. (17) com tempos reduzidos de ventilação mecânica $(M=11 \mathrm{~h})$, quando comparados com o Grupo controle $(\mathrm{M}=28 \mathrm{~h})$.

No nosso trabalho, o tempo médio de ventilação mecânica foi de $94,8 \pm 66,2 \mathrm{~h}$, no Grupo UFC e média de 95,6 \pm 90,2h, no Grupo UFC+UFM.

Estas diferenças no tempo de ventilação mecânica talvez estejam relacionadas com o maior grau de complexidade cirúrgica de nosso Grupo.

Por outro lado, os tempos de permanência em UTIP e de internação hospitalar foram semelhantes nos grupos UFC e UFC + UFM, no nosso estudo (M $=169 \pm 90,6 \mathrm{~h}$ vs $157,8 \pm 150 \mathrm{~h}$ e $\mathrm{M}=14,8 \pm 5,5 \mathrm{~d}$ vs. $14,6 \pm 9,6 \mathrm{~d}$, respectivamente). No entanto, NAIK et al. (7) mostraram tempos de permanência em UTIP e internação hospitalar, menores no Grupo UFM. Os autores comentam que esses resultados devem ser interpretados com cautela porque o número de pacientes foi pequeno e havia diferença significante no peso e grau de complexidade cirúrgica, entre os grupos UFM e controle.

No nosso estudo, a utilização de diálise peritoneal foi semelhante em ambos os grupos, correspondendo a uma incidência de $25 \%$ dos casos, com tempo médio de uso de $81,20 \pm 82,46 \mathrm{~h}$, para 0 Grupo UFC e 69,60 \pm 49,77h, para o Grupo UFC + UFM. Estes dados não puderam ser analisados estatisticamente, em virtude do pequeno número de pacientes ( 5 casos em cada Grupo) e ao tempo real do emprego da diálise, que teve interrupções por problemas técnicos. Acreditamos que, aumentando o número de pacientes em ambos os Grupos, a tendência ao uso de diálise será decrescente.

A HP associada à resistência vascular pulmonar elevada é responsável pela alta morbimortalidade, após correção de defeitos cardíacos, com auxílio da CEC $(30,31)$

Ainda são pouco conhecidos os mecanismos que mantém a HP no período pós-operatório. Estudos recentes sugerem que o tono vascular pulmonar é regulado pela interação de substâncias vasoativas produzidas pelo endotélio vascular $(32,33)$.

A endotelina-1 produzida pelas células do endotélio vascular é um potente agente vasoativo, que participa no mecanismo fisiopatológico da HP. As crianças portadoras de cardiopatias congênitas com HP, apresentam concentrações elevadas de endotelina-1, após finalizada a CEC, seguido de aumento significativo da relação pressão pulmonar sistólica/ pressão arterial sistólica (PPS/PAS) $(34,35)$

Existem protocolos para o controle da pressão arterial pulmonar, após correção de cardiopatias congênitas que incluem: hiperventilação moderada durante a fase de ventilação mecânica, mantendo a $\mathrm{PaO}_{2}>120 \mathrm{mmHg}$, a $\mathrm{PaCo}_{2} \cong 30$ a $35 \mathrm{mmHg}$ e pH $\cong 7,45$ a 7,50 . Os pacientes permaneceram sedados e curarizados até iniciar o desmame do ventilador.

As drogas vasodilatadoras utilizadas são escolhidas conforme o protocolo de cada Instituição. O uso de clorpromazina e prazosin ( $\propto$ bloqueadores), iniciados após CEC, tem mostrado um efeito vasodilatador pulmonar importante e controle de HP.

O tono vascular pulmonar é regulado pelo endotélio vascular, mantendo um equilíbrio entre agentes vasoativos. A endotelina-1 responsável pela HP no $\mathrm{POI}$ pode ser ultrafiltrada durante (UFC) e após CEC (UFM).

Estudos realizados por BANDO et al. (35) demonstraram que o uso da hemodiluição total durante a CEC associada à UFM, em crianças submetidas à correção de má-formações cardíacas com HP, permite eliminar o excesso de água corporal, ultrafiltrar a endotelina-1 e reduzir o número e volume de transfusões de hemoderivados. Os autores concluem que, apesar dos cuidados acima citados, não existe, ainda, uma fórmula mágica para controle de pressão arterial pulmonar.

Por outro lado, fatores hemodinâmicos como a persistência de estenose pulmonar residual ou comunicação intracavitária residual poderão ser responsáveis pela liberação de mediadores vasoativos. A temperatura e o fluxo da CEC também são importantes fatores que aumentam a liberação de endotelina1 e outras citoquinas, induzindo a HP. 
Maluf MA, Mangia C, Bertuccez J, Silva C, Catani R, Carvalho W, Carvalho A, Buffolo E - Estudo comparativo da ultrafiltração convencional e associação de ultrafiltração convencional e modificada na correção de cardiopatias congênitas com alto risco cirúrgico. Rev Bras Cir Cardiovasc 1999; 14 (3): 221-36

No nosso estudo, o Grupo UFC + UFM formado por 20 pacientes, $14(70,0 \%)$ apresentavam HP. Todos os 4 pacientes que faleceram deste grupo, tinham HP.

Dois pacientes portadores de TGA + CIA + HP (5 meses) e "truncus arteriosus" tipo I + HP (11 meses) evoluíram com HP grave (PPS/PAS = 1) faleceram, um por baixo débito cardíaco após arritmia cardíaca e outro por descontinuidade acidental na administração de drogas vasoativas.

Outros 2 pacientes deste Grupo ( $n \div 6$ e n11) faleceram por complicações pulmonares (infecção pulmonar).

No Grupo de UFC, formado por 21 pacientes, $15(71,4 \%)$ tinham HP, 6 pacientes faleceram, sendo que 5 tinham HP. Dentre as causas de óbito, temos: coagulopatia (1 caso), extubação acidental (1 caso), crise de HP (2 casos) e complicações pulmonares (1 caso).

Finalmente, uma criança submetida à correção de origem anômala de ACE apresentou baixo débito cardíaco durante o transporte para a UTIP, falecendo poucas horas depois.

\section{CONCLUSÕES}

- A técnica de UFM foi adaptada com facilidade ao circuito da CEC. Como vantagem aproveita a própria linha da cardioplegia, permitindo, assim, o aquecimento do sangue filtrado e a reposição total do volume do circuito, elevando os níveis do $\mathrm{Ht}$. O maior tempo de canulação da Ao e AD, considerado desvantagem do método, não levou a intercorrências graves durante o procedimento.

- Os métodos de ultrafiltração (UFC e UFC+UFM) foram efetivos na retirada do excesso de líquido do perfusato e do paciente, evitando o edema generalizado e permitindo o fechamento do tórax em condições hemodinâmicas estáveis, independentemente do tempo de CEC.

- No pós-operatório os pacientes submetidos a UFC + UFM não apresentaram diferenças estatisticamente significantes com os pacientes submetidos a UFC isolada, independentemente de idade, peso e complexidade das operações. Estes resultados deverão ser avaliados com cautela, porque se trata de um estudo não randomizado, com número pequeno de pacientes, todos submetidos a UFC.

Maluf MA, Mangia C, Bertuccez J, Silva C, Catani R, Carvalho W, Carvalho A, Buffolo E - Comparative study of standard ultrafiltration and its association with modification for the correction of high surgical risk congenital heart diseases. Rev Bras Cir Cardiovasc 1999; 14(3): 221-36

ABSTRACT: Surgical correction of complex cardiac malformations that require extended extracorporeal circulation (ECC) increase morbidity/mortality due to water retention and systemic inflammatory reaction. The purpose of this study is to compare the immediate postoperative evolution of patients submitted to conventional ultrafiltration (CUF) during ECC and modified ultrafiltration (MUF) after ECC. Forty-one patients submitted to surgical correction of congenital cardiac disease were divided into 2 groups: $\mathrm{G} 1-21$ patients with ages from 15 days to 36 months (median 11 months) and weighing from $3.6 \mathrm{~kg}(\mathrm{M}: 7.27 \pm 3.07)$, operated on between 1996 and 1997 were submitted to CUF; G2 - 20 patients with ages ranging from 9 days to 36 months (median 5.5)

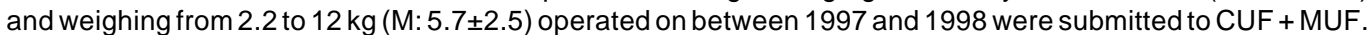
Among the most frequent surgeries were: ventriculoseptoplasty, 15 (36.58) cases; Jatene's surgery, 10 (24.38) cases; correction of septal A-V defect, 7 (17.08) cases, etc. Statistical analysis regarding age, weight and surgical complexity showed similarity between the groups. There were 6 (28.58) deaths in $\mathrm{G} 1$ and 4 (20.08) in $\mathrm{G} 2, \mathrm{p}=0.71$. The mean ultrafiltered volume in $\mathrm{G} 1$ (CUF) was $143.3 \mathrm{ml}$ and $227.0 \mathrm{ml}$ in $\mathrm{G} 2$ (CUF+MUF) $\mathrm{p}<0.001$, showing a statistically significant difference. However, the mean time of mechanical ventilation (G1: 94.8 $\mathrm{h}$ and $\mathrm{G} 2:$ 95.6 $\mathrm{h}, \mathrm{p}=0.97)$, mean time of inotropic drug use ( $\mathrm{G} 1: 145.2 \mathrm{~h}$ and $\mathrm{G} 2: 137.6 \mathrm{~h}, \mathrm{p}=0.85)$, mean time stay in Intensive Care Unit ( $\mathrm{G} 1: 169.6 \mathrm{~h}$ and $\mathrm{G} 2: 157.8 \mathrm{~h}, \mathrm{p}=0.79)$ and mean time of hospital stay ( $\mathrm{G} 1$ : $14.8 \mathrm{~d}$ and $\mathrm{G} 2: 14.6 \mathrm{~d}, \mathrm{p}=0.95$ ) did not show significant differences between the groups. The CUF technique used for more than 8 years on our Service showed similar results when compared with the CUF + MUF association. MUF was efficient regarding removal of body water after ECC without intercurrent episodes due to the method. Possibly a randomized study on a greater number of patients would allow to detect differences between the two methods.

DESCRIPTORS: Heart defects, congenital, surgical. Hemofiltration, methods. Extracorporeal circulation. 
Maluf MA, Mangia C, Bertuccez J, Silva C, Catani R, Carvalho W, Carvalho A, Buffolo E - Estudo comparativo da ultrafiltração convencional e associação de ultrafiltração convencional e modificada na correção de cardiopatias congênitas com alto risco cirúrgico. Rev Bras Cir Cardiovasc 1999; 14 (3): 221-36

\section{REFERÊNCIAS BIBLIOGRÁFICAS}

1 Brull $L-$ Realization de l'ultrafiltration in vivo. Cardiovasc Surg 1928; 99: 1605-7.

2 Alwall $\mathrm{N}-$ In vitro test of dialyses-ultrafilter. Acta Med Scand 1947; 127: 317.

3 Lunderquist A - The efficacy of the dialyses-ultrafilter intended for human use: including a preliminary report of treatment of oedemic patients by means of ultrafiltration. Acta Med Scand 1952; 143: 307-14

4 Romagnoli A, Hocker J, Keatz A, Milan J - External hemoconcentration after deliberate hemodilution. In: Annals of the Annual Meeting of the American Society of Anesthesiologists. Park Ridge, IL, USA, 1976.

5 Darup J, Bleese N, Kalmar P, Lutz G, Pokar H, Polonius $M \mathrm{~J}$ - Hemofiltration during extracorporeal circulation (ECC). Thorac Cardiovasc Surg 1979; 27: 227-30.

6 Magiligan D J Jr. - Indications for ultrafiltration in the cardiac surgical patient. J Thorac Cardiovasc Surg 1985; 89: 183-9.

7 Naik S K, Knight A, Elliott M J - A successful modification of ultrafiltration for cardiopulmonary bypass in children. Perfusion 1991; 6: 41-50.

8 Naik S K, Knight A, Elliott M J - A prospective randomized study of a modified technique of ultrafiltration during pediatric open heart surgery. Circulation 1991; 84: (Suppl III): 422-31.

9 Maluf M A - Experiência com a utilização convencional associada da ultrafiltração modificada na correção cirúrgica de cardiopatias congênitas com auxílio da circulação extracorpórea. [Tese.Livre Docência] São Paulo: Escola Paulista de Medicina da Universidade Federal de São Paulo, 1998

10 Jenkins $K$ J, Newburger J W, Lock J E, Davis R B, Coffman $G A$, lezzoni L I - In hospital mortality for surgical repair of congenital heart defects: preliminary observations of variation by hospital caseload. Pediatrics 1995; 95: 323-30.

11 Kirklin J K \& Barratt-Boyes B G - Cardiac surgery. 2nd ed. New York: Churchill Livingstone, 1993: 78.

12 Groom R C, Aki B F, Albus R A, Hill A, Munuz R, Lefrak E A - Alternative method of ultrafiltration after cardiopulmonary bypass. Ann Thorac Surg 1994; 58: 573-4.

13 Draaisma A M, Hazekamp M G, Frank M, Anes N, Schoof $\mathrm{P} \mathrm{H}$, Huysmans $\mathrm{H} \mathrm{A}$ - Modified ultrafiltration after cardiopulmonary bypass in pediatric cardiac surgery. Ann Thorac Surg 1997; 64: 521-5.

14 Friesen R H, Campbell D N, Clarke D R, Tornabene M A - Modified ultrafiltration attenuates dilutional coagulopathy in pediatric open heart operations. Ann Thorac Surg 1997; 64: 1787-9.

15 larustovskii M B, Il'in $\vee \mathrm{N}$, Abramian $\mathrm{M} \vee$ et al. - The use of modified ultrafiltration in correcting complex congenital heart defects in newborn and nursing infants. Anesteziol Reanimatol 1998; 1: 41-7.

16 Gurbuz A T, Novick W M, Pierce C A, Watson D C Impact of ultrafiltration on blood use for atrial septal defect closure in infants and children. Ann Thorac Surg 1998; 65: 1105-8.

17 Journois D, Israel-Biet D, Pouard P et al. - High-volume, zero-balanced hemofiltration to reduce delayed inflammatory response to cardiopulmonary bypass in children. Anesthesiology 1996; 85: 965-76.

18 Schlunzen L, Pedersen J, Hjortholm K, Hansen O K, Ditlevsen $\mathrm{E}$ - Modified ultrafiltration in pediatric cardiac surgery. Perfusion 1998; 13: 105-9.

19 Darling E, Nanry K, Shearer I, Kaemmer D, Lawsons S - Techniques of pediatric modified ultrafiltration: 1996 survey results. Perfusion 1998; 13: 93-103.

20 Finn A, Naik S, Klein N, Levinsky R J, Strobel S, Elliott $\mathrm{M} J$ - Interleukin-8 release and neutrophil degranulation after pediatric cardiopulmonary bypass. $J$ Thorac Cardiovasc Surg 1993; 105: 234-41.

21 Davies M J, Nguyen K, Gaynor J W, Elliott M J - Modified ultrafiltration improves left ventricular systolic function in infants after cardiopulmonary bypass. J Thorac Cardiovasc Surg 1998; 115: 361-70.

22 Ad N, Snir E, Katz J, Birk E, Vidne B A - Use of the modified technique of ultrafiltration in pediatric openheart surgery: a prospective study. Isr J Med Sci 1996; 32: 1326-31.

23 Brans Y W, Dweck H S, Havis H B et al. - Effects of open heart surgery on the body composition of infants and young children. Pediatr Res 1981; 15: 1024-8.

24 Kirklin J K, Blackstone E H, Kirklin J W - Cardiopulmonary bypass: studies on its damaging effects. Blood Purif 1987; 5: 168-78.

25 Maehara T, Novak I, Wyse R K H, Elliott M J - Perioperative monitoring of total body water by bio-electrical impedance in children undergoing open heart surgery. Eur J Cardiothorac Surg 1991; 5: 258-65.

26 Hodges U M, Berg S, Naik S K, Bower S, Lloyd-Thomas A, Elliott $\mathrm{M} \mathrm{J}$ - Filtration of fentanyl is not the cause of the elevation of arterial blood pressure associated with post-by pass ultrafiltration in children. $J$ Cardiothorac Vasc Anesth 1994; 8: 653-7.

27 Gaynor J W, Tulloch R M R, Owen C H, Sullivan I D, Elliott $\mathrm{M}$ J - Modified ultrafiltration reduces myocardial edema and reverses hemodilution following cardiopulmonary by pass in children. J Am Coll Cardiol 1995; 25: 200-A

28 Elliott $\mathrm{M} \mathrm{J}$ - Ultrafiltration and modified ultrafiltration in pediatric open heart operations. Ann Thorac Surg 1993; 56: 1518-22. 
Maluf MA, Mangia C, Bertuccez J, Silva C, Catani R, Carvalho W, Carvalho A, Buffolo E - Estudo comparativo da ultrafiltração convencional e associação de ultrafiltração convencional e modificada na correção de cardiopatias congênitas com alto risco cirúrgico. Rev Bras Cir Cardiovasc 1999; 14 (3): 221-36

29 Andreasson S, Gotheberg S, Berggren H, Bengtsson A, Eriksson E, Risberg B - Hemofiltration modifies complement activation after extracorporeal circulation in infants. Ann Thorac Surg 1993; 56: $1515-7$.

30 Hopkins R A, Bull C, Haworth S G, De Leval M R, Stark $\mathrm{J}$ - Pulmonary hypertensive crises following surgery for congenital heart defects in young children. Eur $J$ Cardiothorac Surg 1991; 5: 628-34.

31 Bando K, Turrentine M W, Sharp T G et al. - Pulmonary hypertension after operations for congenital heart disease: analysis of risk factors and management. $J$ Thoracic Surg 1996; 112: 1600-9.

32 Vane J R, Anggard E E, Botting R M - Regulatory func- tions of the vascular endothelium. N Engl J Med 1990; 323: 27-36.

33 Cooper C J, Landzberg M J, Anderson T J et al. - Role of nitric oxide in the local regulation of pulmonary vascular resistance in humans. Circulation 1996; 93: 266-71.

34 Komai H, Adatia I T, Elliott M J, De Leval M R, Haworth $S \mathrm{G}$ - Increased plasma levels of endothelin-1 after cardiopulmonary bypass in patients with pulmonary hypertension and congenital heart disease. J Thorac Cardiovasc Surg 1993; 106: 473-8.

35 Bando K, Vijay P, Turrentine M W et al. - Dilutional and modified ultrafiltration reduces pulmonary hypertension after operations for congenital heart disease: a prospective randomized study. $J$ Thorac Cardiovasc Surg 1998; 115: 517-25. 